\title{
Avaliação da microinfiltração de selante oclusal em esmalte de dentes decíduos: efeito de diferentes técnicas de aplicação
}

\author{
- Dayse Andrade Romão Departamento de Cariologia, Universidade Federal de Alagoas, Maceió, AL, Brasil • Marcos Aurélio \\ Bomfim da Silva Departamento de Materiais Dentários, Universidade Federal de Alagoas, Maceió, AL, Brasil • Larissa \\ Silveira de Mendonça Fragoso Departamento de Dentística da Universidade Federal de Alagoas, Maceió, AL, Brasil • \\ Marilia Mattar de Amoêdo Campos Velo Departamento de Dentística, Endodontia e Materiais Odontológicos da Faculdade \\ de Odontologia de Bauru, Universidade de São Paulo, Bauru, SP, Brasil • Fernanda Regina Ribeiro Santos Departamento \\ de Odontopediatria, na Universidade de Pernambuco, Recife, PE, Brasil • Natanael Barbosa dos Santos Departamento de \\ Cariologia, Universidade Federal de Alagoas, Maceió, AL, Brasil
}

RESUMO | Objetivos: Considerando que os dentes decíduos são mais propensos ao desenvolvimento de cárie dentária, este estudo teve como objetivo comparar o efeito de diferentes técnicas de aplicação de selante de fóssulas e fissuras, isoladas ou associadas, na microinfiltração de esmalte em molares de dentes decíduos. Materiais e métodos: dentes molares decíduos foram selecionados ( $\mathrm{n}=8$ ) e receberam os seguintes tratamentos: 1) grupo controle: condicionamento ácido + selante; 2) ar abrasivo + condicionamento ácido + selante; 3) condicionamento ácido + adesivo + selante; 4) ar abrasivo + condicionamento ácido + adesivo + selante. Após o tratamento, os dentes foram imersos em água destilada a $37^{\circ} \mathrm{C}$ por sete dias e então submetidos a termociclagem de 350 ciclos de banhos de água fria $\left(5{ }^{\circ} \mathrm{C}\right)$ e quente $\left(55^{\circ} \mathrm{C}\right)$, com 30 segundos cada exposição. Posteriormente, os dentes foram cobertos com um verniz - deixando exposta apenas a área selada, estendida por $1 \mathrm{~mm}$-, impermeabilizados com resina epóxica e imersos em solução de azul de metileno por 48 horas a $37^{\circ} \mathrm{C}$. Em seguida, os dentes foram seccionados vestíbulo-lingualmente e avaliados microscopicamente (aumento de 40×). Os resultados foram submetidos aos testes de Kruskal-Wallis e de Mann-Whitney ( $<$ o,05). Resultados: os grupos que receberam o adesivo antes da aplicação do selante apresentaram o menor índice de microinfiltração. O grupo que recebeu ar abrasivo mais condicionamento ácido antes do selante mostrou microinfiltração mais extensa. Conclusões: nenhuma das técnicas utilizadas evitou completamente a microinfiltração, entretanto a aplicação de adesivo pode ser a mais indicada.

DESCRITORES | Cárie Dentária; Dente Decíduo; Prevenção Primária.

ABSTRACT | Evaluation of the microleakage of occlusal sealant in deciduous enamel teeth: effect of different application techniques • Objectives: Since deciduous teeth are more prone to the development of dental decay, this study aimed to compare the effect of different strategies for sealant application in pits and fissures, whether isolated or associated, on the enamel microleakage in molars of deciduous teeth. Materials and methods: deciduous teeth molars were selected $(\mathrm{n}=8)$ and received the following treatments: 1) control group: acid etching + sealant; 2) abrasive air + acid etching + sealant; 3) acid etching + adhesive + sealant; 4) abrasive air + acid etching + adhesive + sealant. After treatment, the teeth were immersed in distilled water at $370^{\circ} \mathrm{C}$ for seven days and subjected to thermocycling of 350 cycles of cold $\left(5{ }^{\circ} \mathrm{C}\right)$ and hot $\left(55^{\circ} \mathrm{C}\right)$ baths, for 30 seconds in each exposure. Afterwards, the teeth were covered with varnish - leaving only the sealed area exposed, extended by $1 \mathrm{~mm}$-, waterproofed with epoxy resin and immersed in methylene blue solution for 48 hours at $37^{\circ} \mathrm{C}$. Then, the teeth were buccal-lingually sectioned and microscopically evaluated (40× magnification). The results were subjected to the Kruskal-Wallis and to the Mann-Whitney tests $(\mathrm{p}<0.05)$. Results: the groups that received the adhesive prior to the sealant application have presented the lowest index of microinfiltration. The group that received abrasive air and acid etching before the sealant have showed more extensive microleakage. Conclusions: none of the techniques conducted herein have completely avoided the microleakage, however the adhesive application may be the most indicated technique.

DESCRIPTORS | Dental Decay; Deciduous Teeth; Primary Prevention.

\begin{abstract}
AUTOR CORRESPONDENTE | - Marilia Mattar de Amoêdo Campos Velo Faculdade de Odontologia de Bauru, Universidade de São Paulo • Alameda Dr. Octávio Pinheiro Brisolla, 9-75 Jardim Brasil Bauru, SP, Brasil • 17012-901 E-mail: mariliavelo@yahoo.com.br
\end{abstract}

- Received Aug. 12, 2018 • Accepted Oct. 19, 2018

- Dol http://dx.doi.org/10.11606/issn.2357-8041.clrd.2018.148897 


\section{INTRODUÇÃO}

A dentição decídua apresenta alta suscetibilidade ao desenvolvimento de lesões de cárie. ${ }^{1}$ Esse maior risco pode estar relacionado principalmente à anatomia dos sulcos e das fissuras, como também a experiência anterior de cárie apresentada pelo paciente, à sua história de exposição ao flúor e à presença de biofilme dental. ${ }^{2}$ Um dos tratamentos preventivos para essas lesões é a aplicação de selante, método considerado eficaz para diminuir a incidência e a prevalência de cárie oclusal, com boa relação custo-benefício..$^{3-4} \mathrm{O}$ selante de superfície atua obliterando as fóssulas e fissuras, ${ }^{5}$ restringindo o acúmulo de biofilme e, consequentemente, promovendo uma barreira para a troca de íons metabólicos entre os micro-organismos e o meio, inibindo o processo de desmineralização dental..$^{5-6}$

No entanto, os benefícios desse tratamento dependem diretamente da capacidade de vedação do material, de forma que este deve apresentar aderência à superfície do esmalte, preenchendo as fóssulas e fissuras, e ainda permanecer intacto durante os desafios que acometem o meio bucal. ${ }^{3}$ Algumas condições são determinantes no sucesso dessa estratégia preventiva, destacando-se os hábitos comportamentais do paciente e o adequado isolamento do substrato dentário durante o procedimento. Adicionalmente, a técnica de aplicação é considerada um fator essencial, pois uma adequada adesão, adaptação e penetração do selante ao substrato dental determina a sua durabilidade, enquanto um inadequado selamento marginal aumenta o risco de desenvolvimento de cárie por meio da microinfiltração marginal. ${ }^{6-7}$

De forma geral, a técnica de aplicação de selante de fóssulas e fissuras consiste inicialmente no condicionamento ácido, seguido pela aplicação do produto selador. Entretanto é possível verificar na literatura a existência de diferentes técnicas de preparo para aplicação de selante com o objetivo de diminuir as taxas de microinfiltração. Uma dessas técnicas refere-se ao uso de um agente adesivo previamente à aplicação do selante, formando uma camada intermediária entre o selante e o esmalte dental, que possui propriedades de baixa viscosidade, permitindo uma maior retenção do material e, consequentemente, diminuindo a microinfiltração. ${ }^{7}$

Outra forma que tem sido preconizada é a utilização de ar abrasivo previamente à aplicação do selante. Esse método consiste na aplicação de finos jatos de ar com partículas abrasivas, ocasionando a remoção de detritos e manchas, e fornecendo uma superfície áspera que favorece uma maior retenção do selante. Contudo há na literatura algum debate quanto à eficácia dessa técnica em relação à microinfiltração. ${ }^{8}$ Adicionalmente, é possível observar que não existe trabalho que tenha avaliado o uso do ar abrasivo associado ao adesivo e à técnica convencional.

Assim, o objetivo deste estudo in vitro foi comparar o efeito de diferentes técnicas de aplicação (técnica convencional, ar abrasivo e adesivo) de selante de fóssulas e fissuras na microinfiltração de molares decíduos, comparando os tratamentos quando utilizados de forma individual e associada.

\section{MATERIAIS E MÉTODOS}

Foram utilizados 32 molares decíduos livres de cárie, manchas, trincas e outros defeitos visíveis por meio de lupa estereoscópica. Os dentes foram doados pelo Banco de Dentes da Faculdade de Odontologia da Universidade Federal de Alagoas, com aprovação por Comitê (Processo no oo9850/2009-61), armazenados em timol a $0,1 \%$ e estocados sob refrigeração $\left(4{ }^{\circ} \mathrm{C}\right)$ até o momento de uso no experimento. Após profilaxia com pedra-pomes e água, os 32 dentes foram divididos aleatoriamente em quatro grupos que receberam os seguintes tratamentos: grupo 1 (controle) - condicionamento ácido (ácido fosfórico a 37\%) durante 15 segundos, lavagem, secagem e aplicação do selante (Conseal ${ }^{\circledR}$ 
- SDI, São Paulo, SP, Brasil), usando-se um microbrush, sendo também fotopolimerizados com luz halógena de intensidade $700 \mathrm{~mW} / \mathrm{cm}^{2}$ por 20 segundos (aparelho Optilight Digital ${ }^{\circledR}$ - Gnatus, Ribeirão Preto, SP, Brasil); grupo 2 - aplicação de ar abrasivo (partículas de $50 \mu \mathrm{m}$ ) por 15 segundos, a uma distância de $5 \mathrm{~mm}$ e perpendicularmente à face oclusal, condicionamento ácido, lavagem, secagem e aplicação do selante Conseal ${ }^{\circledR}$ de forma idêntica à do grupo 1; grupo 3: condicionamento ácido por 15 segundos, lavagem, secagem, aplicação do agente de união Stae ${ }^{\circledR}$ (SDI, São Paulo, SP, Brasil), polimerização por 10 segundos e aplicação do selante Conseal ${ }^{\circledR}$ com polimerização de 20 segundos; grupo 4: aplicação de ar abrasivo de forma idêntica à do grupo 2, condicionamento ácido, aplicação do adesivo Stae ${ }^{\circledR}$ e do selante Conseal ${ }^{\circledR}$ de forma idêntica à do grupo 3 .

O sistema adesivo e o selante foram aplicados nos dentes de acordo com as instruções do fabricante. Após a aplicação do selante, os dentes foram imersos em água destilada a $37^{\circ} \mathrm{C}$ por sete dias e, em seguida, submetidos a ciclagem térmica (água fria a $5{ }^{\circ} \mathrm{C}$ e quente a $55{ }^{\circ} \mathrm{C}$, durante 30 segundos em cada banho, num total de 350 ciclos). Posteriormente, foram totalmente cobertos com um verniz, deixando expostos apenas o selante e $2 \mathrm{~mm}$ ao seu redor, sendo então imersos em solução de azul de metileno a $2 \%$ por 48 horas a $37^{\circ} \mathrm{C}$. Decorrido esse período, os dentes foram lavados e secos para a remoção de todo o corante. Cada dente foi seccionado vestíbulolingualmente com um disco diamantado dupla face (KG Sorensen Ind. e Com. Ltd.), e duas secções foram obtidas de cada dente.

Para analisar a microinfiltração do corante na interface selante-esmalte dental, as secções foram avaliadas em um microscópio de luz polarizada (aumento de 40×), por um único examinador. Os escores utilizados foram: $\mathrm{o}=$ nenhuma penetração do corante; 1 = penetração no terço oclusal da interface esmalte-selante; 2 = penetração restrita ao terço médio da interface; 3 = penetração em toda a fissura ${ }^{9}$ (Figura 1).

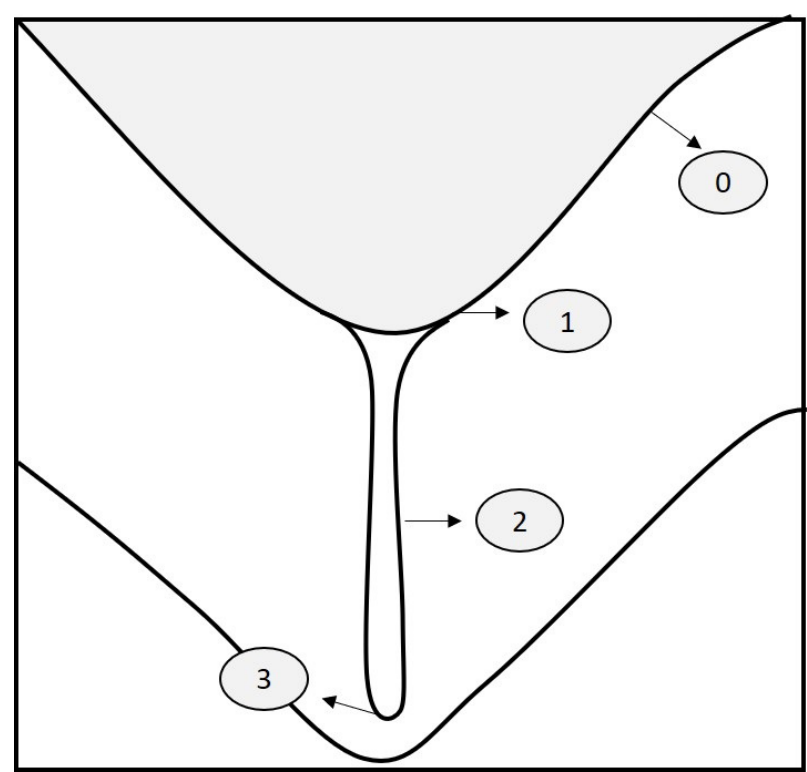

Figura 1 | Esquema representativo dos escores atribuídos para avaliar a microinfiltração do selante.

O software Bioestat 5.0 (Belém, PA, Brasil) foi utilizado para execução da análise estatística. Para comparar a microinfiltração entre os quatro grupos, foi utilizado o teste não paramétrico de KruskalWallis, complementado pelo teste de Mann-Whitney, ao nível de $5 \%$ de significância $(\mathrm{p}<0,05)$.

\section{RESULTADOS}

Os resultados demonstram que as amostras dos grupos 1 e 2 apresentaram maior distribuição entre os escores, com penetração do corante desde no terço oclusal até em toda a fissura, tendo o grupo 2 apresentado maior grau de microinfiltração que todos os outros grupos $(1 \times 2: \mathrm{p}=0,032 ; 2 \times 3$ : $\mathrm{p}=0,005 ; 2 \times 4: \mathrm{p}=0,005)$. Em relação aos grupos 3 e 4, cujas amostras receberam adesivo em uma etapa do tratamento, estes apresentaram menor grau de microinfiltração, sem amostras distribuídas no escore de penetração em toda a fissura, não apresentando 
diferença estatística $(\mathrm{p}=0,15)$. A Tabela 1 expõe as frequências de distribuição das amostras entre os diferentes escores de avaliação de microinfiltração aplicados nesse estudo.

Tabela 1 || Distribuição (n) e frequência (\%) dos escores entre os grupos de tratamento

\begin{tabular}{c|c|c|c|c}
\hline Escores & Grupo 1 & Grupo 2 & Grupo 3 & Grupo 4 \\
\hline 0 & $5(62,5)$ & $0(0,0)$ & $7(87,5)$ & $7(87,5)$ \\
\hline 1 & $2(25,0)$ & $4(50,0)$ & $0(0,0)$ & $0(0,0)$ \\
\hline 2 & $0(0,0)$ & $1(12,5)$ & $1(12,5)$ & $1(12,5)$ \\
\hline 3 & $1(12,5)$ & $3(37,5)$ & $0(0,0)$ & $0(0,0)$ \\
\hline Total & $8(100,0)$ & $8(100,0)$ & $8(100,0)$ & $8(100,0)$ \\
\hline
\end{tabular}

\section{DISCUSSÃO}

O uso de selante de fóssulas e fissuras tem sido considerado um método preventivo da formação de lesões de cárie desde o momento da erupção dos dentes. Entretanto é possível observar que grande parte dos cirurgiões-dentistas não apresentam conhecimento sobre o uso adequado de selantes. ${ }^{10}$ Nesse contexto, pode-se dar destaque às técnicas de aplicação para reduzir a microinfiltração.

Dessa forma, este estudo contemplou o uso de diferentes técnicas e a associação entre elas. Em relação à técnica convencional, utilizada no grupo 1, a maior parte das amostras não apresentou penetração do corante, e em apenas uma amostra o terço médio foi atingido pelo pigmento. Isso demonstra que a técnica convencional, caracterizada pelo condicionamento ácido e pela aplicação de selante, não evitou completamente a microinfiltração, porém é a mais utilizada pelos profissionais. Esses resultados corroboram os dados encontrados em estudo anterior, que também verificou taxas de microinfiltração ao se utilizar a técnica convencional. ${ }^{11}$

Em relação à utilização de ar abrasivo, sua eficácia para evitar microinfiltração é controversa. Nos resultados do presente estudo, a técnica de aplicação de ar abrasivo realizada anteriormente ao condicionamento ácido (método aplicado no grupo 2) foi a que apresentou as maiores taxas de microinfiltração, com penetração do corante em todas as amostras avaliadas e diferença estatística em relação aos demais grupos. Esses resultados divergem de estudos anteriores, realizados em dentes permanentes, que verificaram diminuição das taxas de microinfiltração quando o ar abrasivo é associado ao método convencional e que no seu uso isolado, sem o condicionamento ácido, há aumento da microinfiltração. ${ }^{8,12-13} \mathrm{O}$ mesmo foi verificado em estudo com dentes decíduos ${ }^{14}$, mostrando que essa diferença poderia estar relacionada ao efeito da distância de aplicação do ar abrasivo, que foi efetuada com a metade da distância. ${ }^{14}$ Entretanto, outro estudo demonstrou que não houve diferença entre a técnica convencional e a associação desta com o ar abrasivo. ${ }^{11}$

A inserção de mais uma etapa no selamento, a utilização do adesivo após o condicionamento ácido, foi verificada nos grupos 3 e 4 . No grupo 3 foi possível observar um maior selamento das amostras, quando comparado aos grupos da técnica convencional e do ar abrasivo, sendo que apenas uma de suas amostras apresentou penetração do corante na superfície selada (escore 2). Dessa forma, o adesivo permitiu uma maior vedação, atuando como uma camada intermediária que permite maior adesão e adaptação do selante ao substrato. Os dados sugerem que esse método apresentou a maior viabilidade para a técnica de aplicação de selante, aumentando seu efeito preventivo, o que corrobora dados de estudo in vivo no qual, após cinco anos de aplicação, notou-se que o uso do adesivo aumentou a durabilidade do selante. ${ }^{2}$

Os resultados da avaliação desses métodos, quando conduzidos individualmente, demonstram que o uso do adesivo se sobressai com menores taxas de microinfiltração. Isso poderia explicar os resultados encontrados nos grupos 3 e 4: ambas as amostras receberam o adesivo em uma etapa do tratamento, e essa condição permitiria maior 
proteção contra a microinfiltração. Porém, fazendose a associação de ar abrasivo, adesivo e técnica convencional, etapas realizadas no grupo 4 , foi possível verificar que não houve efeito aditivo de retenção com a utilização do ar abrasivo, pois uma amostra também apresentou penetração do corante (escore 2). Entretanto, como pode ser observado, esses dados foram semelhantes àqueles encontrados no grupo 3 (adesivo e condicionamento ácido), em que ocorreu apenas microinfiltração restrita ao terço médio da interface, sugerindo que não há necessidade de utilização do ar abrasivo no processo de aplicação do selante e que este não diminuiu as taxas de microinfiltração.

Os resultados deste estudo sugerem que a técnica de aplicação do selante é uma das etapas necessárias para a aumentar a durabilidade do material e que a aplicação prévia de adesivo mostrou resultados promissores. Entretanto, deve-se ponderar o acréscimo de uma etapa nesse processo de aplicação, em termos de custo-benefício na saúde pública, além de se considerar os hábitos comportamentais do paciente. Outro fator a ser verificado, necessariamente, é a viabilidade da associação dessas técnicas também em estudos in situ ou in vivo.

\section{CONCLUSÃO}

Os resultados sugerem que, independentemente da técnica utilizada, não houve como evitar completamente a microinfiltração, sendo a aplicação de adesivo previamente ao selamento a técnica mais indicada. Entretanto, posteriores estudos in situ e in vivo ainda são necessários.

\section{REFERÊNCIAS}

1. Velo MM, Tabchoury CP, Romão DA, Cury JA. Evaluation of low fluoride toothpaste using primary enamel and a validated pH-cycling model. Int J Paediatr Dent. 2016;26(6):439-47.

2. Feigal RJ, Donly KJ. The use of pit and fissure sealants. Pediatr Dent. 2006;28(2):143-50.
3. Borsatto MC, Thomaz MY, Contente MM, Gomes-Silva JM, Mellara TS, Galo R, et al. Bonding agent underneath sealant: shear bond strength to oil-contaminated. Braz Dent J. 2010;21(1):50-4.

4. Mickenautsch S, Yengopal V. Validity of sealant retention as surrogate for caries prevention - a systematic review. PLOS One. 2013;8(10):e77103.

5. Chestnutt IG, Chadwick BL, Hutchings S, Playle R, Pickles T, Lisles C, et al. Protocol for "Seal or Varnish?" (SoV) trial: a randomised controlled trial to measure the relative cost and effectiveness of pit and fissure sealants and fluoride varnish in preventing dental decay. BMC Oral Health. 2012;12:51.

6. Braz AK, Aguiar CM, Gomes AS. Evaluation of the integrity of dental sealants by optical coherence tomography. Dent Mater. 2011;27(4):e6o-4.

7. Nogourani MK, Janghorbani M, Khadem P, Jadidi Z, Jalali S. A 12-month clinical evaluation of pit-and-fissure sealants placed with and without etch-and-rinse and self-etch adhesive systems in newly-erupted teeth. J Appl Oral Sci. 2012;20(3):352-6.

8. Lupi-Pégurier L, Muller-Bolla M, Bertrand MF, Fradet T, Bolla M. Microleakage of a pit-and-fissure sealant: effect of air-abrasion compared with classical enamel preparations. J Adhes Dent. 2004;6(1):43-8.

9. Grande RH, Ballester R, Singer JM, Santos JF. Microleakage of a universal adhesive used as a fissure sealant. Am J Dent. 1998;11(3):109-13.

10. Govindaiah S, Bhoopathi V. Dentists' levels of evidence-based clinical knowledge and attitudes about using pit-and-fissure sealants. J Am Dent Assoc. 2014;145(8):849-55.

11. Duangthip D, Lussi A. Effects of fissure cleaning methods, drying agents, and fissure morphology on microleakage and penetration ability of sealants in vitro. Pediatr Dent. 2003;25(6):527-33.

12. Ellis RW, Latta MA, Westerman GH. Effect of air abrasion and acid etching on sealant retention: an in vitro study. $\mathrm{Pe}$ diatr Dent. 1999;21(6):316-9.

13. Courson F, Renda AM, Attal JP, Bouter D, Ruse D, Degrange M. In vitro evaluation of different techniques of enamel preparation for pit and fissure sealing. J Adhes Dent. 2003;5(4):313-21.

14. Knobloch LA, Meyer T, Kerby RE, Johnston W. Microleakage and bond strength of sealant to primary enamel comparing air abrasion and acid etch techniques. Pediatr Dent. 2005;27(6):463-9. 